\title{
A framework for effective collaboration : a case study of collaboration in nursing education in the Western Cape, South Africa.
}

\author{
Felicity M. Daniels, Thembisile D. Khanyile
}

School of nursing, University of Western Cape, South Africa

A fundamental purpose of mergers between higher education institutions (HEIs) in 2002 was to enable sharing of scarce resources between more advanced universities and those historically disadvantaged by the apartheid system of the South African Government. A common teaching platform for undergraduate nursing education in the Western Cape was established in 2005, in line with the transformation of the higher education system, as a collaborative initiative between three universities.In order to evaluate the common teaching platform, Stuffelbeam's context, input, process, product (CIPP) research model was employed. A sample of 108 participants was selected through stratified purposive sampling, and included three deputy vice-chancellors, three deans, three heads of department, 18 lecturers and 81 students. Semi-structured interviews were held with the staff members, whilst the students participated in focus group interviews. Open-ended questions informed by literature and the CIPP evaluation model were developed and used to guide the interviews. This enabled the researcher to obtain a rich description of the participants' experiences. The data were analysed inductively. The results revealed that the main purpose of collaboration was not achieved due to the lack of a common understanding of the concept of collaboration and its purpose; a lack of readiness to collaborate and a lack of sharing of resources. A framework for effective collaboration was developed based on the results.

\section{Introduction}

Historically, educational institutions have collaborated for a variety of reasons. For instance, in the early 1980 s, a major rationale for collaboration was to strengthen resources, whereby an institution with more or more advanced resources would collaborate with another with fewer resources. In the 1990s, the notion of building capital had a major influence on collaboration initiatives. Whilst capital can be conceptualized as human, material, physical, social and cultural, most international collaborative projects focused mainly on the human resources element to build capacity. This notion of collaboration, as capacity building, has dominated the international higher education arena for the past century, and countries including 
those in Africa are seen as good 'laboratories' for international research. Literature abounds with positive experiences, as well as the advantages of and lessons learned from such collaborative initiatives. However, most of these experiences and lessons have been published by international partners and very few by their African counterparts.

Types of Collaboration

\section{Keywords: Collaboration}

Common teaching platform Undergraduate nursing programme Higher education According to Dowling et al. (2004), collaboration is a process in which autonomous actors interact through formal or informal negotiations, jointly creating rules and structures governing their relationships and ways to act or make decisions on the issues that brought them together. It is a process involving shared norms and mutually beneficial interaction (Dowling et al., 2004). There are different types of collaboration, characterised by the level of political will and the complexity of the implementation process. Where the political will is high, the implementation process tends to be less complex. Voluntary collaboration exists where institutions are highly motivated to collaborate with each other, usually in academic programme areas and where it can be managed at faculty level with little or no regional governance resources required (Leatt and Pretorius, 2004). Institutionally-driven collaboration exists where the senior leadership of two or more institutions is keen to collaborate, with low levels of interest from other levels of staff.

These collaboration opportunities are identified at institutional planning level or through a regional planning review, and would require full regional governance resources. A third type of collaboration is that of international partnerships, which are usually institutionally driven, with a limited number of staff being knowledgeable about and interested in its functions. Finally, externally driven collaboration is mainly characterised by a low level of political will and a highly complex implementation process. This type of collaboration is mainly identified by the Ministry, and typically presented to the institution as a policy imperative. The parties involved are usually not ready to collaborate and perceive this as being imposed upon them, and may resist attempts to collaborate (Leatt, 2003).

The National Plan for Higher Education (Department of Education, 2001) describes the purpose of collaborative efforts within regions in South Africa. It was envisaged that collaboration in higher education would result in institutions in a region remaining separate but combining their expertise, efforts and infrastructural resources in the development and delivery of higher education programmes. The purpose of such collaboration was to improve diversity in the programmes offered by universities and to reduce the costs within the region. It was predicted that the collaborative use of academic expertise, human resources and infrastructure across universities would strengthen programmes and contribute to efficient use of facilities and resources for teaching, learning and research (Department of Education, 2001). 
In 2002, as part of the restructuring exercise, the Minister of Education directed that the University of Cape Town (UCT) and the University of Stellenbosch (US) no longer offer undergraduate nursing programmes leading to registration with the South African Nursing Council as a professional nurse. Instead, undergraduate nursing should be offered by the University of the Western Cape (UWC) and a new institution, the Cape Peninsula University of Technology (CPUT), which resulted from a merger of the Cape and Peninsula Technikons (Department of Education, 2002, p. 17).

The Cape Higher Education Consortium (CHEC), which represents the five institutions of higher learning in the Western Cape, identified undergraduate nursing education as a priority for collaboration, and argued that the need for qualified nurses in the region required that the universities of Cape Town and Stellenbosch support UWC in the offering of the undergraduate programme. Consequently the CHEC submitted a counter-proposal to the Minister of Education to operate undergraduate nursing education in the province under a common teaching platform (CTP) model of collaboration, with UWC and CPUT as the enrolling institutions. This proposal was accepted by the Minister in 2004 (CHEC, 2006). The collaborating institutions crafted a Memorandum of Understanding (MoU) which contained guidelines and a framework to guide the operationalisation of the CTP. National and regional nurse shortages and the closure of undergraduate programmes at the universities ofCape Town and Stellenbosch necessitated an increase in the student intake at UWC from 120 first-year students in 2003 to 320 in 2004 and beyond.

Based on challenges institutions experienced with transformation, the Minister of Education commissioned the establishment of a ministerial committee in 2008 to report on the progress of transformation in South Africa. According to the final report, institutional understanding of transformation, discrimination and social cohesion was in line with the White Paper's vision and framework for transformation. The committee found in most instances that institutions had put policies in place to deal with transformation issues. It could thus be said that in legal and regulatory terms, higher education institutions were in a good position. There were concerns, however, that transformation compliance might have been a paper exercise in some instances, although the report acknowledges the significant strides that a number of institutions have made with regard to broader transformation in terms of institutional culture and epistemological change (Soudien, 2008).

In the Western Cape, collaboration among the three universities to transform nursing education continued despite certain challenges. CPUT withdrew from participating on the CTP and continued to offer their own nursing programmes. The effects of transformation on the delivery of the nursing programme and on the patient, as well as possibilities for improvement, were yet to be established.

This article shares the lessons learned from this collaborative initiative, and proposes a framework for effective collaboration based on the strengths and challenges of the collaboration. 


\section{Purpose of the Study}

After five years of collaboration between universities in the Western Cape and the numerous challenges experienced, it was necessary to evaluate the common teaching platform model of collaboration.

\section{Methodology Research Design}

An evaluation research design was used to gain detailed insight into various participants' experiences of collaborating on the CTP. Stufflebeam's Context, Input, Process and Product (CIPP) model was used as a framework for this study. Whilst the purposes of evaluations vary, Stufflebeam argued that evaluations should not be limited to determining whether the objectives of a programme have been reached, but should lead to managing and improving programmes (Madaus et al., 1986). This was also the purpose of the evaluation of the CTP.

\section{Sampling}

The sample $(\mathrm{n}=108)$ was obtained through stratified purposive sampling and included 27 academics from the three institutions (UWC, UCT and US) and 81 students from year one to year four of the programme. The academics included three deputy vice-chancellors, three deans, three heads of department and 18 lecturers. The inclusion criterion was that participants must have been involved in the design, development and implementation of the CTP.

\section{Data Collection}

Semi-structured in-depth interviews were conducted with the academics to gain detailed insight into their experiences of the collaboration. The assumption was that as they were knowledgeable about the collaboration, their perspectives and experiences of the CTP would be meaningful and enhance the success of the study. Focus group interviews with registered nursing students allowed the researcher to obtain many different views within a short period. Eight focus groups were conducted with male and female students, two per year level 1 to 4 .

\section{Data Analysis}

The data were analysed inductively which enabled the researcher to make sense of the data without imposing pre-existing expectations on the phenomenon (Patton, 2002). The inductive data analysis process described by Thomas (2003) was used. The audiotapes were listened to several times before the semi structured interviews and focus group interviews were transcribed verbatim. The transcriptions were carefully 
read to become familiar with the content. An Nvivo software package, version 8, was used to code the text segments that formed meaningful units into labelled categories. These categories were refined to reduce overlapping and redundancy. Some categories where combined or linked, when their meanings were similar. The categories which captured the key aspects of the themes in the raw data were regarded as the major themes which were relevant to the research objectives.

The broad research questions examined were:

What was the participant's understanding of the concept of collaboration?

In their view, was the context conducive to collaboration?

What were their experiences of the implementation of the collaborative initiative?

Based on the findings, a framework for effective collaboration was developed.

\section{Research Ethics}

The proposal received ethical clearance from the Ethics Committee, the project was registered (Project No. 06/4/5) and the proposal approved by the UWC. The Dean of Research at the university, the Director of the School of Nursing and the Nursing Academic Board granted permission for the research to be conducted.

The purpose of the study and its potential benefits for nursing education was explained to the participants. Verbal and written informed consent was obtained from all participants. Participants gave informed consent for the use of an audiotape recorder. Participation in the study was voluntary, and participants could withdraw from the study at any time with no consequences whatsoever. Anonymity was assured throughout and after the study period.

\section{Results}

A number of themes relating to participants' understanding and experiences of the collaborative process emerged from the data.

\section{Lack of Readiness to Collaborate}

Lecturers and deputy vice-chancellors shared the sentiment that the universities were not ready for collaboration due to differing philosophies and conflicts of interest. A head of department reported that one of the universities withdrew at the last minute from delivering agreed upon modules in the undergraduate programme. This increased the workload of staff at enrolling institutions. The same department head also alluded to staff and students' lack of readiness to collaborate and the lack of resources for implementing the CTP, which resulted in numerous operational challenges.

\section{Reason for the Collaboration Not Well Understood}

Lecturers felt that political transformation in South Africa was relatively young and that people needed time to adjust. One head of department suggested that people did not understand transformation in the context of rebuilding the nation, whilst another 
reported that senior staff had resigned because they did not believe in or want to be part of the change. When the beliefs of staff regarding change are not aligned with those of the organisation, it can result in differences in the level of commitment to the change process between senior management and the rest of the staff. Providing a clear rationale for collaboration and 'unmistakable steps' driven by people in leadership positions is important (Langlands, 2012, p. 29). Furthermore, Hay et al. (2001) argue that in institutional change, such as mergers, organisational goals must be regarded as more important than individual needs. One of the deputy vice-chancellors referred to this when he cautioned on existing tensions between the universities: "When it comes to universities there is a tension between collaboration and competition ... but if we can look at ourselves as part ofthe system and the public good role ofuni-versities... then we will begin to see the potential benefits of collaboration."

Whilst maintaining respect for institutional identity was regarded as important, the need to move beyond separate institutional priorities and make concessions for the benefit of the whole, was identified as crucial in a partnership of five higher education institutions in Cornwall (Langlands, 2012).

\section{Top-Down Approach Used to 'Force' Collaboration}

The heads of department felt that the goals and vision of the common teaching platform were forced on them. They were of the opinion that senior management of the universities was more accepting of the goals and visions of the common teaching platform. This was corroborated by the deputy vice-chancellors, who felt that senior management was more willing to participate, with less will and enthusiasm at the head of department level. The views of the heads of department and the deputy vice-chancellors support the idea that the common teaching platform was an institutionally-driven collaboration, characterised by willingness among senior leadership of the institutions to collaborate and less willingness among staff at lower levels. As one of them asserted: 'It was like a forced marriage. If it's forced, it won't work.' This comment also signifies a top-down approach. Lessons from collaboration between two higher education institutions in Amsterdam were that whilst senior staff must be key in driving the collaboration, the process must adopt an inclusive approach (Langlands, 2012). Other issues highlighted by the heads of department, which justified their lack of political will to collaborate, concerned the differing institutional cultures.

\section{Lack of Understanding of the Concept of Collaboration}

The deans of the universities felt that a common understanding of collaboration between partners on the common teaching platform was lacking. One of the deans reported that there was always tension, which was expressed in a derogative way. She reported that despite the arrangement being new to everyone, every mistake was 
blown out of proportion by colleagues. The memorandum of understanding, which was the guiding framework for the collaboration, was also not understood and interpreted in the same way by all the partners.

\section{Administrative Challenges}

The lack of integration of administrative systems was another reason why the universities were not ready for change, according to the deputy vice-chancellors. The heads of department and lecturers referred to difficulties which lecturers at the partner universities experienced in accessing the administrative system of the enrolling university. These included issues such as the marks administration systems and the fact that the academic terms of the three institutions did not coincide, among others. This resulted in an increased workload for the co-ordinators in the programme at the enrolling university. Studies conducted by Connolly et al. (2007) and Cragg et al. (2003) in the United Kingdom and Canada respectively also found that administrative issues can become challenging if the logistics are not worked out beforehand.

\section{Lack of sharing of resources}

All participants referred to the fact that sharing of resources and expertise across the platform did not occur as was intended. A student reported: 'I think to a very large extent a lot of us did not benefit from the collaboration as much as we could've. I came to do nursing and I want to be the best nurse that I can be. I used all the resources that were handed to me in this course to do the best that I could. but if more resources were given, less excuses were given for why they weren't available, I could've been much better than I am.'

Based on his concern for nursing education, Lord Willis, Chair of the Education Commission of the Royal College of Nursing announced: "If there are ways in which we can make the education experience for nurses better, we need to seize them with both hands - for the benefit of the student nurse and patients everywhere" (Willis, 2012).

The lack of sharing of expertise and resources across universities means that the opportunity to strengthen the nursing education programme for the region was missed, which according to the Department of Education (2001) was the core purpose of the collaboration. To illustrate how the concept of sharing of expertise was undermined, a lecturer from the enrolling university reported that 'partner institutions don't use their expertise; they employ part-time lecturers to teach on the $C T P^{\prime}$. One of the lecturers mentioned that students viewed the lecturers of two of the partner institutions as guest lecturers. Fundamental to the process of collaboration are the concepts of sharing of scarce resources and the fostering of a sense of partnership and interdependency between collaborating universities. 


\section{Lack of Diversity in Student Profile}

One of the department heads alluded to the fact that diversity in the student profile at the enrolling university, which was expected to result from the enrolment of students who would have potentially registered at the partner universities, did not occur. Applications to the three institutions were not pooled and uninformed applicants erroneously applied to the partner institutions. As a result there were no substantial changes in the student profile at the enrolling university.

\section{Lack of Commitment to the Collaborative Initiative}

Some lecturers felt there was a lack of commitment to collaborate, and that the staff at the enrolling university was burdened by the heavy administrative load resulting from the collaboration. A Dean alluded to the fact that the collaboration was lucrative for their institution in terms of the payment received for participation, but admitted that the CTP was not a high priority on their institution's agenda. A deputy vice-chancellor highlighted the need to conduct a cost-benefit analysis of the CTP, in order to establish whether this collaborative initiative indeed reduced the costs in the region, as was anticipated by the Department of Education.

\section{Discussion}

The results revealed that the implementation process was experienced as challenging by all participants, due to differences in the understanding of the concept of collaboration. Langlands (2012) suggests that effective collaboration is dependent on mutual trust between the parties, especially during the period of negotiation. McKeown (2002) suggests that uncertainty about the future causes anxiety, which results in negative behaviours which may obstruct the achievement of the goal. This uncertainty might be another reason why staff of the partner university resigned from their jobs.

The aim of collaboration on the CTP was to strengthen capacity in the Nursing Department at UWC to carry out the mandate of being one of two enrolling universities for undergraduate nursing in the Western Cape. It was also aimed at maintaining the autonomy of universities in the region whilst bringing together their expertise and resources. It was evident from the interviews with the deans, heads of department and lecturers that the general feeling was that the goals of collaboration on the CTP were not met. Furthermore, collaboration on the CTP was viewed as a financial burden on the enrolling university, but as a financial gain for the partner institutions.

Langlands (2012) and Hay et al. (2001) suggest that successful collaborative efforts are dependent on a shared vision of and strong commitment to the process. It is not 
viable to commit to a process without a belief in and common vision for the change. Molzahn and Purkis (2004) also identified differing organisational cultures and philosophies as a challenge for merger and collaboration attempts, as was found in this study. Hay et al. (2001) cautioned that merging should not be viewed as a marriage between equal partners, alluding to predictable differences in institutional cultures and philosophies. Wyngaard and Kapp (2004) recognised that in the context of mergers in higher education in South Africa, institutions had no choice regarding merger partners nor were they prepared for the merger process. However, department heads would have been prepared if they had been trained in managing the transformation process, as suggested by Kavanagh and Ashkanasy (2006; in Reddy, 2007). 
FRAMEWORK FOR EFFECTIVE COLLABORATION

COMMUNICATION

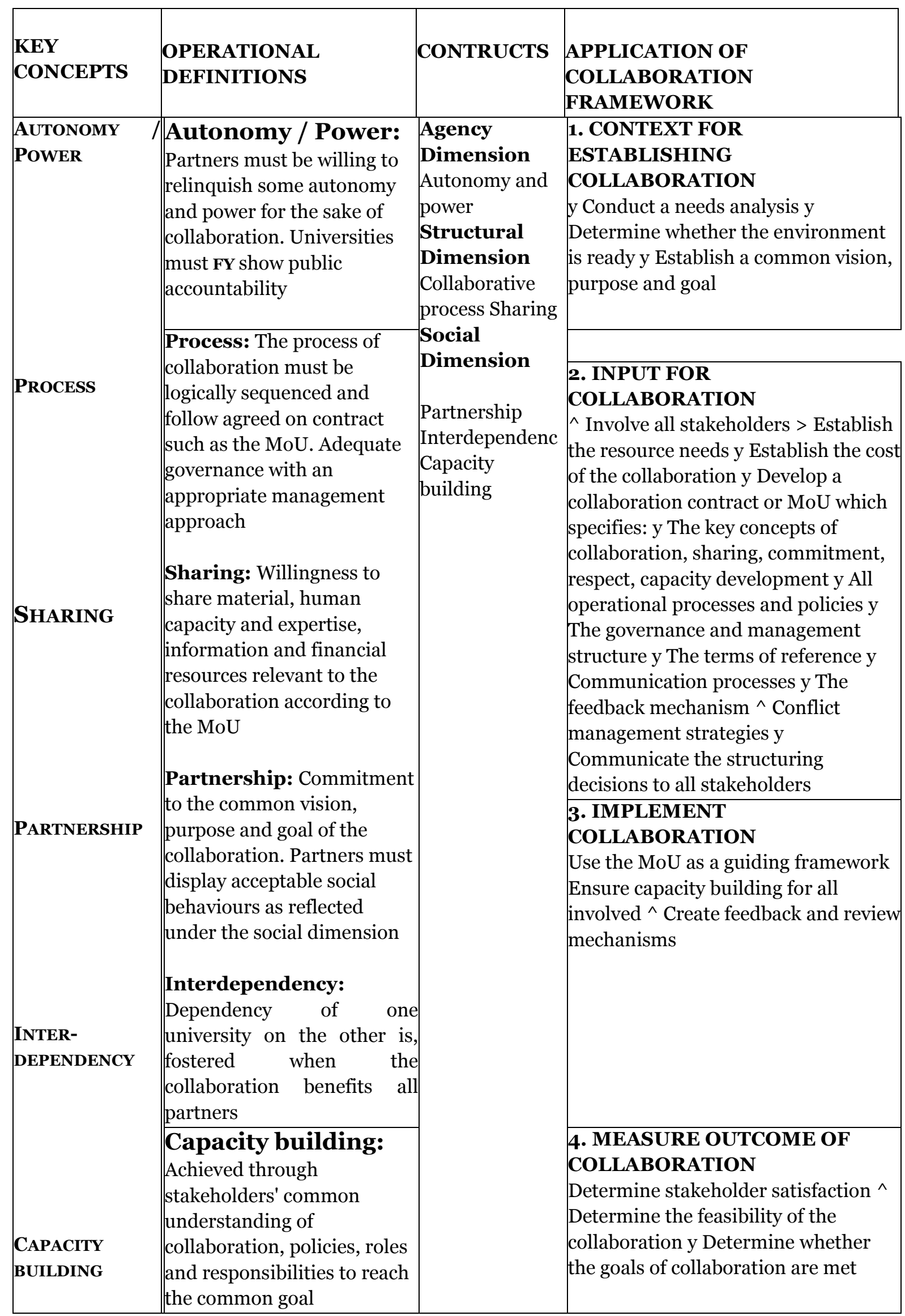




\section{COLLABORATION}

Fig. 1. A framework for effective collaboration.

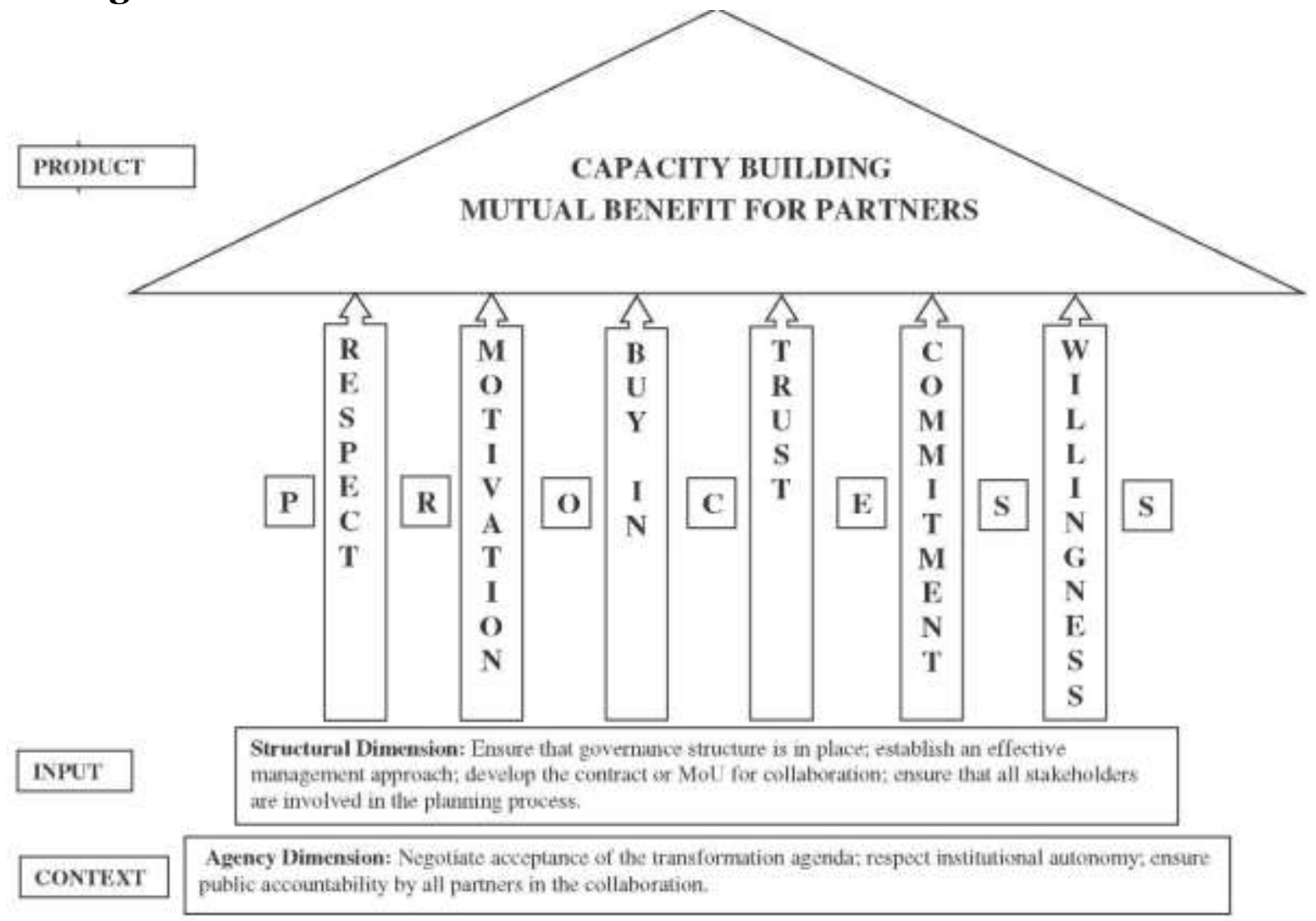

Fig. 2. Application of a framework for effective collaboration.

A conceptual framework is defined as a group of concepts that are broadly defined and systematically organised to provide a focus, rationale, and a tool for the integration and interpretation of information (Mosby, 2009). A conceptual framework is usually expressed abstractly through word models, and is the conceptual basis for many theories, such as communication theory and general systems theory. Conceptual frameworks also provide a foundation and organisation for the educational plan in schools of nursing.

The following key concepts, which posed challenges for collaboration, were derived from the themes and are presented under the following constructs:

i)

gency dimension The concept of institutional autonomy was highlighted self-governance and independence of universities despite their participation in the collaborative initiative. Regional collaboration was aimed at participating universities maintaining their autonomy but bringing together their expertise and resources. However, the collaboration was negatively affected by a lack of willingness and readiness by the universities to participate. The reason for the collaboration was also not well 
understood by all, and a top-down approach to collaboration was reported. The findings of the study also revealed that universities continued to apply their institutional perspective in their interpretation and application of the memorandum of understanding.

ii)

tructural dimension A collaborative process and sharing were central concepts. It was important that the process of collaboration was driven by an adequate governance structure and an effective management approach as well as a well-developed contract or memorandum of understanding. The process of collaboration was intended to comprise a series of actions aimed at meeting the goals of regional collaboration on a CTP for undergraduate nursing in the Western Cape. The rationale for collaboration was however not clearly understood. This led to several challenges, including a general lack of resources for the CTP and a lack of sharing between institutions - both of which are linked to the equity principle of improving the distribution of South Africa's limited resources. Sharing was also identified as one of the basic tenets of collaboration according to the National Plan for Higher Education (Department of Education, 2001). Several other challenges arose because the administration systems of the participating institutions were not synchronised. iii) Social dimension The social dimension refers to mutual behaviours expected of institutions to ensure that the collaboration is successful, including concepts of partnership, interdependency and capacity development. According to Powell and Glendinning (2002), partnerships facilitate the sharing of expertise and best practice in a way that would not have been possible if organisations worked independently. Partnerships also foster acceptable social behaviours and commitment between partners. As referred to earlier, there was a lack of willingness to collaborate, and a lack of sharing of expertise and resources between institutions. Interdependency refers to the benefits of the collaborative efforts for each partner. Interdependency is therefore fostered when there is a sense of partnership and sharing and an understanding that the collaborative initiative benefits all partners. Each university perceived the CTP as beneficial to their own needs and not to the common goal. According to the Africa Centre for Health Population and Studies (2008), capacity building refers to the process by which individuals develop and/or enhance their skills to organise systems, resources and knowledge, as reflected in their abilities (individually and collectively), in order to perform functions, solve problems and set and achieve objectives. The aim of the CTP was to strengthen the capacity in the nursing department at UWC to enable them to be one of two enrolling universities for undergraduate nursing in the Western Cape. However, due to the lack of 
sharing of expertise and institutional resources, the enrolling institution did not experience the intended capacity development.

\section{Conclusion}

Collaboration is a complex phenomenon, the definition of which remains vague or highly variable. Furthermore, the balance between autonomy and the expected interplay between social systems poses complex challenges for implementation of externally driven collaborative efforts. Despite its elusiveness, its essence continues to be sought after as a means of improving working relationships and patient outcomes. This lack of clarity has resulted in the term 'collaboration' being used in a variety of inappropriate ways in both the research and practice settings. For example, it is often considered synonymous with other modes of interaction, such as cooperation or compromise. Unfortunately, confusion over the meaning of collaboration has hindered its usefulness as a variable in studies which attempt to evaluate its effectiveness. This ambiguity may also account for the view of the participants in this study that the goals of the CTP were not met.

\section{Acknowledgements}

The authors acknowledge the funding contribution of the Atlantic Philanthropies which made the study possible.

\section{References}

Africa Centre for Health Population and Studies, 2008. Capacity building policy. Retrieved from http://www.africacentre.ac.za/CapacityBuildingPolicy/tabid/108/Default.aspx. Cape Higher Education Consortium, 2006. Integrated planning framework and memorandum of understanding: a CTP for undergraduate nursing in the Western Cape. (Retrieved from http://www.chec.ac.za/Nursing/Final Mo plus link to Appendices- Version 2, revised at 1 January 2006.pdf).

Connolly, C., Jones, C., Jones, N., 2007. Managing collaboration across further and higher education: a case in practice. Journal of Further and Higher Education 31 (2), 159-169.

Cragg, C.E., Doucette, S., Humbert, J., 2003. Ten universities, one program. Successful collaboration to educate nurse practitioners. Nurse Educator 28 (5), 227-231.

Department of Education, 2001. National Plan for Higher Education. Government Printers, Pretoria.

Department of Education, 2002. Transformation and restructuring: a new institutional landscape for higher education. Government Gazette No. 23549, 21 June 2002. Government Printers, Pretoria. 
Dowling, B., Powell, M., Glendinning, C., 2004. Conceptualising successful partnerships. Health and Social Care in the Community 12 (4), 309-317. http://dx.doi.org/ 10.1111/j.1365-2524.2004.0050.

Hay, H.R., Fourie, M., Hay, J.F., 2001. Are institutional combinations, mergers or amalgamation the answer? An investigation into staff perceptions. South African Journal of Higher Education 15 (1), 100-108.

Langlands, A., 2012. Collaborations, Alliances and Mergers in Higher Education. Higher Education Funding Council for England . (www.hefce.ac.uk/media/hefce/content/pubs/2012/201206/12_06.pdf).

Leatt, J., 2003. Improvements in regional co-operation: the case of the Cape Higher Education Consortium South Africa. Retrieved from http://siteresources.worldbank. org/INTAFRREGTOPTEIA/Resources/james_leatt.pdf.

Leatt, J., Pretorius, T., 2004. Regional collaboration and the transformation of higher education in South Africa. Paper prepared for the European Higher Education Society (EAIR) Conference on Knowledge Society Crossroads, Barcelona (Retrieved from http://www.chec.ac.za/introduction/Barcelona.pdf).

Madaus, G.F., Scriven, M., Stufflebeam, D.L., 1986. Evaluation Models: Viewpoints on Educational and Human Services Evaluation. Kluwer-Nijhoff Publishing, Boston/ The Hague/Dordrecht/Lancaster.

McKeown, R., 2002. Managing change, reorienting education involves managing change: ESD Toolkit, Version 2. Retrieved from http://www.esdtoolkit.org/discussion/change. htm.

Molzahn, A.E., Purkis, M.E., 2004. Collaborative nursing education programs: challenges and issues. Nursing Leadership 17 (4), 41 -55.

Mosby, Inc., 2009. Mosby's Pocket Dictionary of Medicine, Nursing \& Health Professions. Mosby/Elsevier, St. Louis, Mo.

Patton, M.Q., 2002. Qualitative Evaluation and Research Methods, 3rd ed. Sage Publications, California.

Powell, M., Glendinning, C., 2002. In: Glendinning, C., Powell, M., Rummery, K. (Eds.), Partnerships, New Labour and the Governance of Welfare. The Policy Press, Bristol, pp. 51 -66.

Reddy, R.B., 2007. Knowledge Management - Tool For Business Development. Himalaya Publishing House, New Delhi.

Soudien, C., 2008. Final report of the ministerial committee on transformation and social cohesion and the elimination of discrimination in public higher education institutions. Retrieved from www.che.ac.za/.../CHE_response_to_MinisteriaLCommittee_

Report_Dec2009.pdf.

Thomas, D.R., 2003. A General Inductive Approach for Qualitative Data Analysis. School of Population Health, University of Auckland.

Willis, Lord, 2012. We Aim to Ensure Nurses Get the Best Education and Training. NursingTimes.net . (Retrieved from www.nursingtimes.net/we-aim-to-ensurenurses-get-the-best-education-and-training). 
Wyngaard, A., Kapp, C., 2004. Rethinking and re-imagining mergers in further and higher education: a human perspective. South African Journal of Higher Education 18 (1), 185-201. 